\title{
Anti-Ischemia Drugs have no Effect on the In Vivo Metabolism of ATP by RBC in Normotensive Restrained Rats ${ }^{\#}$
}

\author{
Pollen K.F. Yeung*, Julie Dauphinee, Krista Simonson and Thera Gouzoules
}

Pharmacokinetics \& Metabolism Laboratory, College of Pharmacy, Dalhousie University, Halifax, Nova Scotia, B3H 3J5, Canada

\begin{abstract}
The objective is to determine the effect of anti-ishemia agents on metabolism of adenosine-5'-triphosphate (ATP) in red blood cell (RBC) in a normotensive rat model. Male Sprague Dawley (SD) rats weighing between 300 - 400 $\mathrm{g}$ were used. Each rat received either saline (control), or $5 \mathrm{mg} / \mathrm{kg}$ of diltiazem (DTZ), losartan, amlodipine or dipyridamole by subcutaneous injection (sc) twice daily for 5 doses. Blood samples were collected using a "Stopping Solution" from each rat at time 0 (before the last dose), and sequentially after over 6 hours following the last dose via an indwelling carotid artery catheter. In addition, hemodynamic recordings were collected throughout the experiment. Concentrations of ATP and other purine nucleotides in the RBC were determined by a validated HPLC. Data between groups were analyzed by ANOVA and paired t-test, and differences between groups considered significant when $\mathrm{p}<0.05$. The results showed that the concentrations of ATP and the other purine nucleotides in RBC of the rats treated with the anti-ischemia drugs were not different from the control rats (Table 1). The concentrations of ATP and guanosine-5'-triphosphate (GTP) were higher towards the end of the experiment, but the increase was significant only after amlodipine and losartan $(\mathrm{p}<0.05)$. The increase of ATP and AMP concentrations correlated with decrease of diastolic blood pressure (DBP) in the rats. The study concluded that the anti-ischemia drugs tested in the current study have no effect on RBC concentrations of ATP in the restraining rat model.
\end{abstract}

Keywords: ATP, purine nucleotides, metabolism, biomarkers, anti-ischemia drugs, diltiazem.

\section{${ }^{\#}$ The paper was presented in part at the 110th Annual Meeting of American Society of Clinical Pharmacology and Thera- peutics, National Harbor (Washington, DC), MD, March 18 - 21, 2009.}

\section{INTRODUCTION}

Over the last 30 years, considerable progress has been made in drug therapy for chronic ischemic heart disease (IHD). Most pharmacologic therapies act by reducing myocardial oxygen consumption, increasing coronary blood flow, improving ventricular relaxation, dilating collateral vessels, and reducing peripheral resistances. Despite "optimal" medical therapy, a large fraction of patients continue to present with angina and myocardial ischemia [1]. It is recommended that treatment strategies should include modifying risk factors such as diet, weight loss, regular exercise, smoking cessation and adequate control of hypertension, diabetes, and hyperlipidemia [2].

Diltiazem (DTZ), losartan, amlodipine and dipyridamole are clinically proven effective cardiovascular agents for ischemic heart disease and have considerable potential for treatment and prevention of atherosclerosis and stroke [3-5]. While most of these agents are efficacious in lowering blood pressure (BP), their effects on heart rate (HR) are less decisive [6-8]. This may be related to their differences in the mechanism of action and their inherent inhibitory effects on neurohormone activation.

*Address correspondence to this author at the College of Pharmacy, 5968 College Street, Burbidge Building, Dalhousie University, Halifax, NS, B3H 3J5, Canada; Tel: 902-4943845; Fax: 902-4941396;

E-mail: Pollen.Yeung@Dal.Ca
The importance of adenosine and adenosine-5'triphosphate (ATP) concentration in regulating many biological functions has long been recognized, especially for their effects on the cardiovascular system [9-11]. It is known that adenosine and ATP are key factors in regulating of coronary blood flow [12-14], inhibiting platelet aggregation [15], protecting myocardium $[11,16]$, neuromodulation [1719], modulating tissue necrosis [20], ischemic preconditioning $[21,22]$, immunomodulation [10], and perhaps other functions (e.g. pain mediation), which maintain the homeostasis of the cardiovascular system. It has been shown that patients with effort angina and essential hypertension have altered adenosine metabolism compared to normal individuals [23-25], and that plasma concentrations of adenosine increase in patients with congestive heart failure (CHF) [26], which could be a physiologic response to heart failure and help to reduce the severity of the disease [27]. Thus it has been postulated that adenosine and ATP may be used as sensitive biomarkers to quantify myocardial and endothelial ischemia [28-30], a surrogate for post-exercise hypotension [31] and for monitoring therapeutic effects of anti-ischemia drugs [32-35].

There are evidences to suggest that many anti-ischemia agents may increase RBC concentrations of ATP. Previous studies from our laboratories have shown that DTZ increased RBC concentrations of ATP in an exercise rat model and zebrafish model [36, 37], and that RBC concentrations of ATP may be sensitive biomarkers for cardiovascular protec- 
tion $[31,35]$. It is possible that anti-ischemia agents may affect metabolism of ATP in RBC either directly or indirectly which may affect their safety and efficacy. This paper evaluates for the first time the hemodynamic effects of four commonly used anti-ischemia agents and their effects on RBC concentrations of ATP and other adenine nucleotides using a previously described rat model in vivo [38].

\section{MATERIALS AND METHODOLOGY}

DTZ was received as gift from Biovail Corp. (Mississauga, ON, Canada), losartan from Merck \& Co Canada (Point Claire, QC, Canada), amlodipine from Pfizer Canada Inc. (Point Claire, QC, Canada), and dipyridamole from Boehringer Ingelheim (Burlington, ON, USA). Other chemicals were reagent grade and used as such from the stock bottles. The animal study protocol was approved by the Dalhousie University Committee on Laboratory Animals (UCLA). Male Sprague-Dawley (SD) rats, weighing between 300 - $400 \mathrm{~g}$ were purchased from Charles River Laboratories (Wilmington, MA, USA) and allowed to acclimatize for one week in the Dalhousie Carleton Animal Care Center and have access to food and water ad libitum. An indwelling catheter made of silastic ${ }^{\circledR}$ tubing (PE-50, Dow Corning Corp., MI, USA) was implanted into the right carotid artery of each animal under general anaesthesia as described previously [39]. After recovery from the surgery (24 - $48 \mathrm{~h}$ ), each rat received either saline (control $\mathrm{n}=14$ ) or $5 \mathrm{mg} / \mathrm{kg}$ of DTZ $(\mathrm{n}=14)$, losartan $(\mathrm{n}=7)$, amlodipine $(\mathrm{n}=7)$, or dipyridamole $(\mathrm{n}=7)$ by subcutaneous injection (s.c.) twice daily (bid) for 5 doses ( $n=7-14$ per group). This dosage has been shown to induce appreciable hemodynamic effects in rodents in previous study [38, 40], and subcutaneous injection is accurate and more amenable for multiple dosing. Hemodynamic variables (SBP, DBP and HR) were continuously recorded via the intra-vascular catheter after the last dose and up to $6 \mathrm{~h}$ using a Transpac II disposable transducer (Abbott Laboratories, IL, USA) coupled to a Siemens hemodynamic monitor (Sirecust 400) and chart recorder (Siredoc) (Erlangen, FRG) while the rats were kept in a restrainer as previously described (Fig. 1) [38, 41]. Blood samples (0.3 $\mathrm{mL}$ ) were collected using a "Stopping Solution" for measurement of RBC concentrations of ATP, adenosine-5'diphosphate (ADP), adenosine-5'-monophosphate (AMP) and GTP by a validated HPLC assay [36] from each rat at 0 (before the last dose), 0.25, 1, 2, 3, 4, 5 and $6 \mathrm{~h}$ after the last dose via the carotid artery catheter. The blood loss was immediately replenished by the same volume of Ringer's Solution after each collection. In addition, hemodynamic recordings were collected throughout the experiment. Data were analysed by ANOVA and Student's paired t-test, and differences between groups or between samples considered significance when $\mathrm{p}<0.05$. In addition, correlation between hemodynamic variables and concentrations of adenine nucleotides in RBC was evaluated and the relationship considered significant at $\mathrm{p}<0.05$ (Minitab ${ }^{\circledR}$ Inc., Release 15.1, State College, PA, USA).

\section{RESULTS}

Under the described experimental condition (Fig. 1), DBP declined gradually towards the end of the experiment, but the systolic blood pressure (SBP) and heart rate (HR) remained steady in the control rats (Fig. 2). The hemody- namic effects of the anti-ischemia drugs studied were different qualitatively and quantitatively. Since the details of their hemodynamic effects have been reported [40], only a brief account of their difference is summarized here. The average blood pressure lowering effect measured over the 6 hours compared to the control rats were greatest for amlodipine (SBP $-22 \%$ and DBP -28\%), followed by losartan (SBP $10 \%$ and DBP $-17 \%)(\mathrm{p}<0.05$ for all the values), and then diltiazem (SBP $-2.2 \%$ and DBP $-4 \%$ ) ( $\mathrm{p}<0.05$ for DBP). Contrarily, dipyridamole increased SBP significantly $(+6 \%$, $\mathrm{p}<0.05$ ) without an apparent effect on DBP. Compared to control rats, the HR lowering effect averaged over the 6 hours was significant after amlodipine $(-5 \%)$ and diltiazem $(-6 \%)(\mathrm{p}<0.05)$. In contrast, losartan increased HR by $6 \%$ $(\mathrm{p}<0.05)$ and dipyridamole had no significant effect on HR $(+2 \%)$.

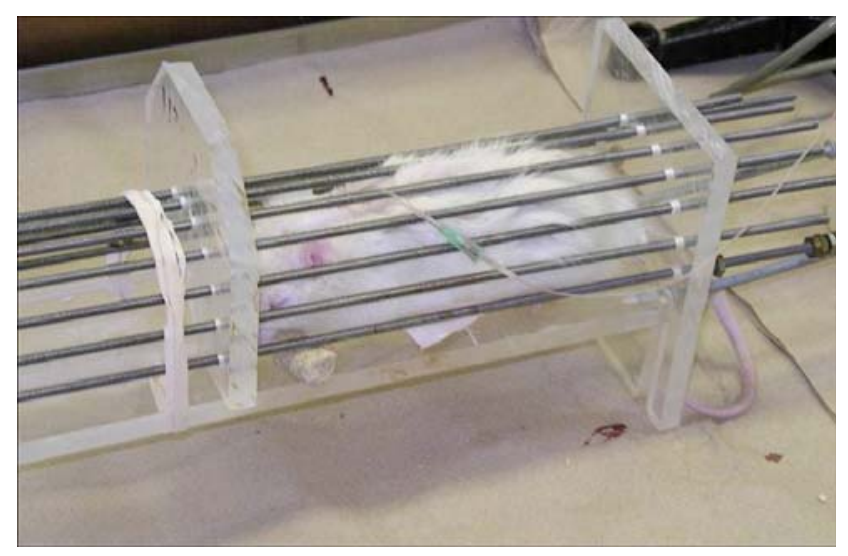

Fig. (1). Restraining rat model.

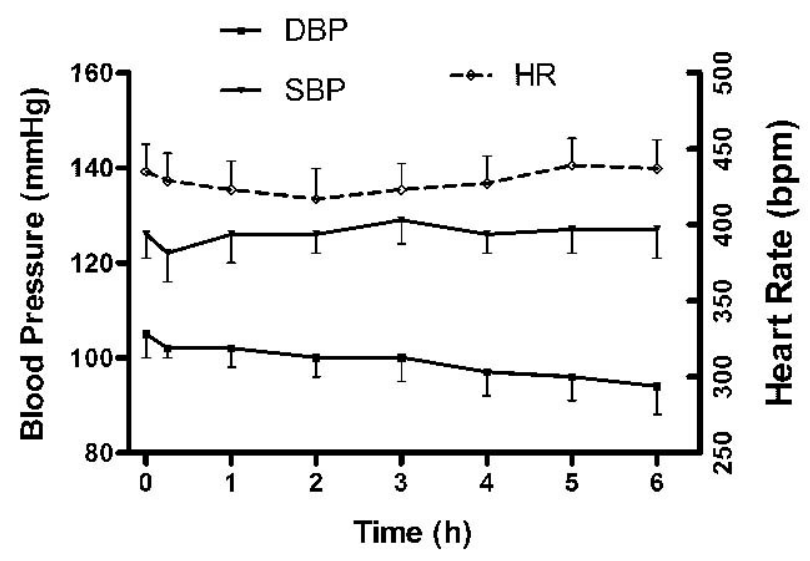

Fig. (2). Hemodynamic effects in control rats (data represent mean \pm SEM).

The average concentration of ATP, ADP, AMP and GTP in $\mathrm{RBC}$ from the control rats determined over the 6-hour experiment was $0.95 \pm 0.48,0.53 \pm 0.18,0.31 \pm 0.39$ and $0.098 \pm 0.043 \mathrm{mM}$, respectively (Table 1). There was a tendency for the purine nucleotide concentrations to increase towards the end of the experiment, however, but only the increase in GTP concentrations was statistically significant $(\mathrm{p}<0.05)$ (Fig. 2). There were no differences in the average RBC concentrations of ATP and other purine nucleotides measured over the $6 \mathrm{~h}$ between the control rats and the rats 
Table 1. RBC Concentrations of ATP and other Adenine Nucleotides

\begin{tabular}{|c|c|c|c|}
\hline Biomarkers/Treatment & Average over 6 hours & Before the experiment (Time 0) & At the end of 6 hours (Time 6) \\
\hline ATP control $(\mathrm{mM})$ & $0.95 \pm 0.48^{\mathrm{a}}$ & $0.85 \pm 0.76$ & $1.04 \pm 0.77$ \\
\hline ATP DTZ (mM) & $0.92 \pm 0.69$ & $0.85 \pm 0.76$ & $1.04 \pm 0.77$ \\
\hline ATP amlodipine (mM) & $0.94 \pm 0.25$ & $0.74 \pm 0.21$ & $1.08 \pm 0.22 *$ \\
\hline ATP losartan (mM) & $0.90 \pm 0.28$ & $0.76 \pm 0.29$ & $1.04 \pm 0.28^{*}$ \\
\hline ATP dipyridamole (mM) & $0.94 \pm 0.50$ & $0.80 \pm 0.38$ & $1.16 \pm 0.39$ \\
\hline ADP control (mM) & $0.53 \pm 0.18$ & $0.53 \pm 0.22$ & $0.55 \pm 0.28$ \\
\hline $\operatorname{ADP}$ DTZ $(\mathrm{mM})$ & $0.60 \pm 0.34$ & $0.62 \pm 0.30$ & $0.62 \pm 0.49$ \\
\hline ADP amlodipine (mM) & $0.82 \pm 0.081^{* *}$ & $0.69 \pm 0.097$ & $0.84 \pm 0.18$ \\
\hline ADP losartan (mM) & $0.75 \pm 0.12$ & $0.77 \pm 0.19$ & $0.75 \pm 0.14$ \\
\hline ADP dipyridamole (mM) & $0.40 \pm 0.085$ & $0.52 \pm 0.21$ & $0.42 \pm 0.087$ \\
\hline AMP control (mM) & $0.31 \pm 0.39$ & $0.25 \pm 0.26$ & $0.33 \pm 0.43$ \\
\hline AMP DTZ $(\mathrm{mM})$ & $0.42 \pm 0.47$ & $0.53 \pm 0.64$ & $0.38 \pm 0.45$ \\
\hline AMP amlodipine (mM) & $0.27 \pm 0.067$ & $0.22 \pm 0.073$ & $0.30 \pm 0.13$ \\
\hline AMP losartan (mM) & $0.22 \pm 0.058$ & $0.24 \pm 0.074$ & $0.22 \pm 0.059$ \\
\hline AMP dipyridamole (mM) & $0.048 \pm 0.020$ & $0.11 \pm 0.083$ & $0.048 \pm 0.036$ \\
\hline GTP control $(\mathrm{mM})$ & $0.098 \pm 0.043$ & $0.081 \pm 0.043$ & $0.11 \pm 0.059 *$ \\
\hline GTP DTZ (mM) & $0.11 \pm 0.073$ & $0.088 \pm 0.063$ & $0.13 \pm 0.10^{*}$ \\
\hline GTP amlodipine (mM) & $0.13 \pm 0.022$ & $0.11 \pm 0.034$ & $0.18 \pm 0.069$ \\
\hline GTP losartan (mM) & $0.088 \pm 0.044$ & $0.075 \pm 0.024$ & $0.094 \pm 0.045$ \\
\hline GTP dipyridamole (mM) & $0.075 \pm 0.027$ & $0.048 \pm 0.023$ & $0.11 \pm 0.045^{*}$ \\
\hline
\end{tabular}

${ }^{\mathrm{a}}$ Data are mean $\pm \mathrm{SD}$.

$* \mathrm{p}<0.05$ vs Time 0

$* * \mathrm{p}<0.05$ vs Control.

treated with the anti-ischemia agents, except for ADP concentration which was higher in the amlodipine treated rats $(\mathrm{p}$ $<0.05$ ) (Table 1). While the RBC concentrations of ATP in the drug treated rats at the end of the experiment increased significantly from $0.74 \pm 0.21$ to $1.08 \pm 0.22 \mathrm{mM}$ and from $0.76 \pm 0.29$ to $1.04 \pm 0.28 \mathrm{mM}$ for amlodipine and losartan, respectively, the increase was not significant from the rats treated with DTZ or dipyridamole. Effects of the antiischemia drugs on RBC concentrations of GTP were also mix. They were significantly higher at the end of the experiments, however, only in the control, or DTZ and dipyridamole treated rats, but not in the rats treated with losartan or dipyridamole (Table 1). Furthermore the gradual decline of DBP in the control rats during the experiment correlated with the steady rise of RBC concentrations of the purine nucleotides (Fig. 3).

\section{DISCUSSION}

The anti-ischemia drugs evaluated in the current study differed considerably for their hemodynamic effects which were mainly attributed to their inherently different pharmacokinetic and pharmacdynamic properties. The calcium channel antagonists DTZ and amlodipine lowered BP and
HR, whereas losartan lowered BP but increased HR, and dipyridamole increased SBP but had no effect on DBP or HR in the rat model. These agents also differed in their duration of action such that the hemodynamic effects were more prolonged after amlodipine and losartan than after DTZ or dipyridamole [40].

As reported in our previous communications and by other laboratories, the RBC have high concentrations of adenine nucleotides in the $\mathrm{mM}$ range $[35,36,42]$. It is very interesting to note that there was a tendency for the nucleotide concentrations in the RBC to increase towards the end of the experiment, which was accompanied by a decrease in DBP, but not SBP or HR (Fig. 2). A significant correlation was obtained between the ATP or AMP concentrations and DBP ( $r=-0.878$ for ATP, and -0.707 for AMP, $p<0.05$ for both), but not with ADP or GTP in the control rats. Similar correlations were also observed for the drug treated rats. This suggests that RBC concentrations of ATP and AMP may be linked to the decline of DBP. A similar relationship between DBP and RBC concentrations of ATP was also found in rats after exercise [31]. The mechanism for the decrease of DBP is not known, although it could be attributed to reduced energy utilization secondary to lack of physical activity as the 

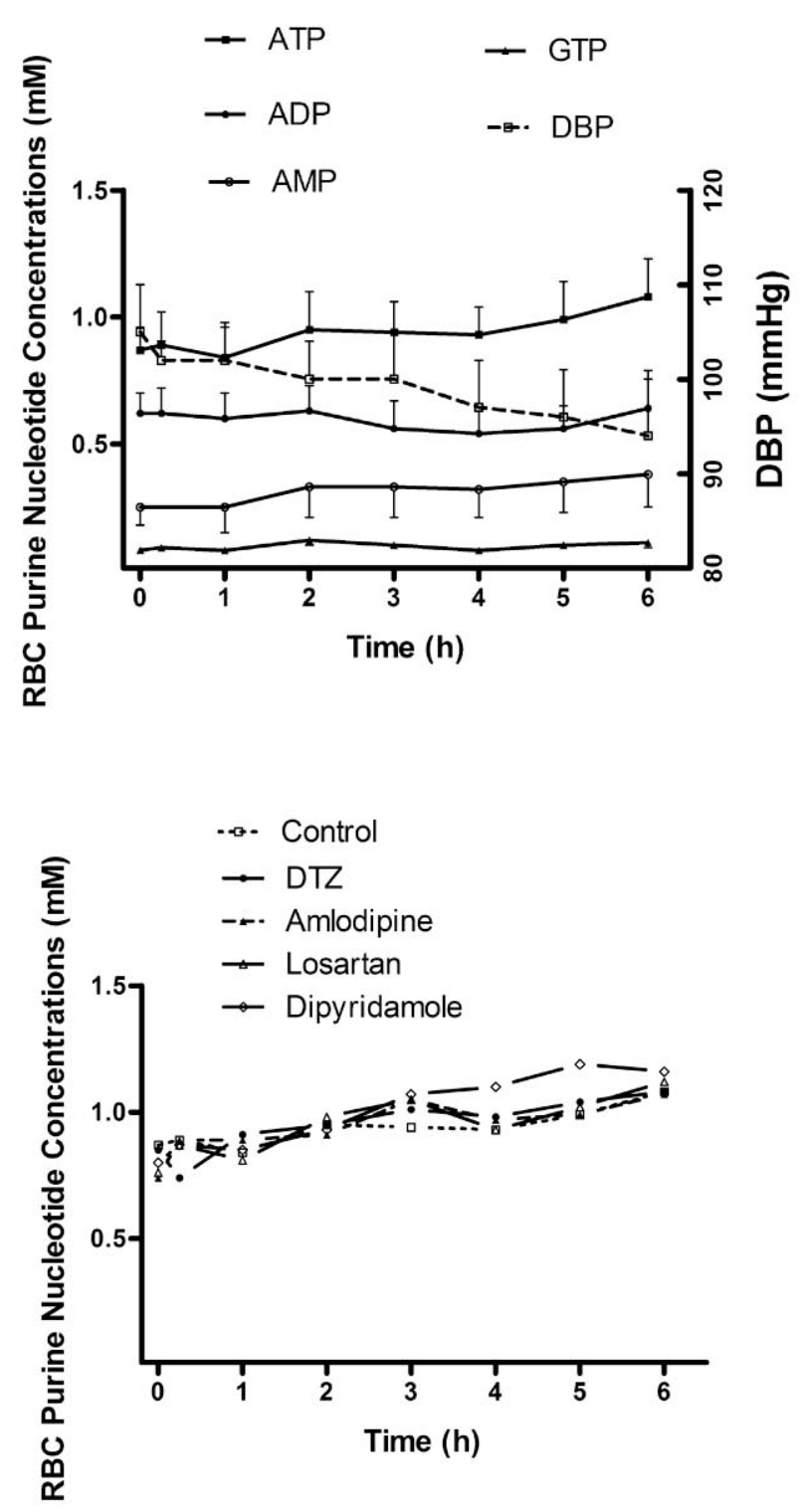

Fig. (3). RBC concentrations of purine nucleotides (Note: only ATP concentrations are shown in the bottom figure).

rats were kept in the restrainer for the entire experiment. It could also partly attributable to the blood loss (approximately $2.5 \mathrm{~mL}$ ) during the experiment as a result of serial blood sample collection. However, there was no difference in the hemodynamic profiles or RBC concentrations of purine nucleotides when we attempted to collect $50 \%$ more of the blood over the span of the 6-hour experiment (approximately $4 \mathrm{~mL}$ in total). Thus further investigation of the relationship between hemodynamic changes in particular DBP, energy metabolism and RBC concentrations of adenine nucleotides using hypertensive rat model and under exercise condition when demand of energy is increased are warranted. This will further enhance our understanding if ATP concentrations in the RBC are surrogates for cardiovascular protection.

We have found consistently in most rats that the concentrations of ADP and AMP were about $40-60 \%$ and $10-$ $30 \%$, respectively, of the ATP concentrations. On the other hand, the GTP concentrations were typically about $10 \%$ of the ATP concentrations (Table 1). Since none of the antiischemia agents tested in the current study significantly affect the RBC concentrations of the purine nucleotides in the rodent model (Table 1) the hemodynamic effects of these anti-ischemia agents are likely not linked directly to their effects on the concentrations of the purine nucleotides in the RBC. Nevertheless, there was a significant increase of ATP concentrations towards the end of the experiment in rats treated with losartan and amlodipine. On the contrary, a significant increase in GTP concentrations was found after DTZ and dipyridamole, and also in the control rats (Table 1). This would imply that there may be an indirect effect of the antiischemia agents on the nucleotide concentrations. Some of the anti-ischemia agents investigated in the current study are substrates/inhibitors of the ATP-binding cassette family of drug transporters in RBC which may affect RBC concentrations of the nucleotides directly [43-46]. Although the significance of concentrations of the different nucleotides in the $\mathrm{RBC}$ is not clear, it is possible that their ratios could change during ischemia when ATP may be broken down to ADP and AMP in RBC in vivo. It has been shown that ATP is released from human $\mathrm{RBC}$ and myocardium in response to a brief period of hypoxia which is broken down to ADP and AMP in vitro $[47,48]$. Similar break down may also occur for GTP although little is known of the physiologic functions of this nucleotide in the RBC and how they may be different from ATP. It is also interesting to know that RBC concentrations of GTP are much higher in the fish (20 - 50\% of ATP) compared to the rodents $[49,50]$. The species difference may be attributed to genetic background and environmental factors which are inherently different between aquatic species and rodents. While there is no direct evidence to indicate a similar event may also occur in vivo, the idea of RBC being an oxygen sensor as suggested by other workers needs further investigation [51, 52]. It is known that $\mathrm{RBC}$ is capable of releasing increased amounts of ATP as oxygen content falls and its haemoglobin becomes desaturated [47]. It has been hypothesized that $\mathrm{RBC}$ may sense tissue oxygen requirements when they travel through the microcirculation and release vasodilatory compounds such as ATP that enhance blood flow in hypoxic tissues [52]. The released ATP would help to increase blood supply to the tissue and preserve an optimum balance between oxygen supply and demand, thereby modulating the concentrations of tissue ATP within the cardiovascular system. Such a mechanism would eliminate the requirement for a diverse network of sensing sites throughout the vasculature, and should provide a more efficient means of appropriately matching oxygen supply with demand. Thus drugs which effect energy metabolism can have an indirect effect on metabolism of ATP in the RBC, particularly under ischemia and stressful condition. This was supported indirectly by an earlier observation that exercise increased RBC ATP concentrations in rats, particularly in those treated with DTZ [36]. If this hypothesis is proven correct, $\mathrm{RBC}$ concentrations of purine nucleotides and their ratios may be used as surrogate biomarkers for management of cardiovascular diseases which would be an exciting topic with important clinical relevance for further studies.

\section{CONCLUSION}

The anti-ischemia drugs investigated in the current study do not affect RBC concentrations of purine nucleotides di- 
rectly. The decline in DBP towards the end of the experiment may be attributed to reduced energy consumption in a restraining environment, and linked to increased RBC concentrations of ATP and AMP, but not attributed directly to the drug effect.

\section{ACKNOWLEDGEMENT}

Supported in part by a grant-in-aid from CIHR/NSHRF/PEF Regional partnership Program.

\section{REFERENCES}

[1] Marzilli, M.; Affinito, S.; Focardi, M. Changing scenario in chronic ischemic heart disease: therapeutic implications. Am. J. Cardiol., 2006, 98(5A), 3J-7J.

[2] Raza, J.A.; Reinhart, R.A.; Movahed, A. Ischemic heart disease in women and the role of hormone therapy. Int. J. Cardiol., 2004, 96 (1), 7-19.

[3] Basile, J.N.; Chrysant, S. The importance of early antihypertensive efficacy: the role of angiotensin II receptor blocker therapy. $J$. Hum. Hypertens, 2006, 20(3), 169-75.

[4] Grossman, E.; Messerli, F.H. Calcium antagonists. Prog. Cardiovasc. Dis., 2004, 47(1), 34-57.

[5] Hegge, K.A. Antiplatelet agents for prevention of recurrent ischemic stroke. S. D. Med., 2009, 62(1), 15-7.

[6] Bang, L.E.; Wiinberg, N.; Wachtell, K.; Larsen, J.; Olsen, M.H.; Tuxen, C.; Hildebrandt, P.R.; Ibsen, H. Losartan versus atenolol on 24-hour ambulatory blood pressure. A LIFE substudy. Blood Press, 2007, 16(6), 392-7.

[7] Chern, C.M.; Hsu, H.Y.; Hu, H.H.; Chen, Y.Y.; Hsu, L.C.; Chao, A.C. Effects of atenolol and losartan on baroreflex sensitivity and heart rate variability in uncomplicated essential hypertension. $J$. Cardiovasc. Pharmacol., 2006, 47(2), 169-74.

[8] Chakrabarti, S.; Freedman, J.E. Dipyridamole, cerebrovascular disease, and the vasculature. Vasc. Pharmacol., 2008, 48(4-6), 1439.

[9] Moens, A.L.; Claeys, M.J.; Timmermans, J.P.; Vrints, C.J. Myocardial ischemia/reperfusion-injury, a clinical view on a complex pathophysiological process. Int. J. Cardiol., 2005, 100(2), 179-90.

[10] Ingwall, J.S. Energy metabolism in heart failure and remodelling. Cardiovasc. Res., 2009, 81(3), 412-9.

[11] Cohen, M.V.; Downey, J.M. Adenosine: trigger and mediator of cardioprotection. Basic Res. Cardiol., 2008, 103(3), 203-15.

[12] Jeremias, A.; Filardo, S.D.; Whitbourn, R.J.; Kernoff, R.S.; Yeung, A.C.; Fitzgerald, P.J.; Yock, P.G. Effects of intravenous and intracoronary adenosine 5 '-triphosphate as compared with adenosine on coronary flow and pressure dynamics. Circulation, 2000, 101(3), 318-23.

[13] Oxhorn, B.C.; Cheek, D.J.; Buxton, I.L. Role of nucleotides and nucleosides in the regulation of cardiac blood flow. AACN Clin. Issues, 2000, 11(2), 241-51.

[14] Tune, J.D.; Richmond, K.N.; Gorman, M.W.; Feigl, E.O. Control of coronary blood flow during exercise. Exp. Biol. Med., (Maywood), 2002, 227(4), 238-50.

[15] Gerlach, E.; Becker, B.F.; Nees, S. Formation of Adenosine by Vascular Endothelium: A Homeostatic and Antithrombogenic Mechanism? Gerlach, E.; Becker, B. F. Eds. The Topic and Perspectives in Adenosine Research. New York NY, Springer-Verlag, 1987, vol. 87, pp. 309-320.

[16] Obata, T. Adenosine production and its role in protection against ischemic and reperfusion injury of the myocardium. Nippon Yakurigaku Zasshi, 2002, 119(5), 273-9.

[17] Burnstock, G. Purinergic signalling: past, present and future. Braz. J. Med. Biol. Res., 2009, 42(1), 3-8.

[18] Leonelli, M.; Torrao, A.S.; Britto, L.R. Unconventional neurotransmitters, neurodegeneration and neuroprotection. Braz. J. Med. Biol. Res., 2009, 42(1), 68-75.

[19] Ralevic, V. Purines as neurotransmitters and neuromodulators in blood vessels. Curr. Vasc. Pharmacol., 2009, 7(1), 3-14.

[20] Burnstock, G. Purinergic signaling and vascular cell proliferation and death. Arterioscler. Thromb. Vasc. Biol., 2002, 22(3), 364-73.

[21] Donato, M.; Gelpi, R.J. Adenosine and cardioprotection during reperfusion--an overview. Mol. Cell Biochem., 2003, 251(1-2), 153-9.
[22] Das, M.; Das, D.K. Molecular mechanism of preconditioning. IUBMB Life, 2008, 60(4), 199-203.

[23] Tykarski, A.; Gluszek, J.; Banaszak, F. Value of oxypurines and uric acid in plasma, renal excretion of oxypurines and uric acid as well as plasma adenosine deaminase and AMP deaminase activity with essential hypertension (in Polish). Pol. Arch. Med. Wewn., 1993, $89,223-229$.

[24] Duthie, G.; Beattie, J.; Arthur, J.; Franklin, M.; Morrice, P.; James, W. Blood antioxidants and indices of lipid peroxidation in subjects with angina pectoris. Nutrition, 1994, 10, 313-316.

[25] Yeung, P.; Buckley, S.; Hung, O.R.; Pollak, P.T.; Barclay, K.D.; Feng, J.D.; Klassen, G.A. Effect of diltiazem on plasma concentrations of oxypurines and uric acid. Ther. Drug Monit., 1997, 19, 286-291.

[26] Funaya, H.; Kitakaze, M.; Node, K.; Minamino, T.; Komamura, K.; Hori, M. Plasma adenosine levels increase in patients with chronic heart failure. Circulation, 1997, 95(6), 1363-5.

[27] Kitakaze, M.; Minamino, T.; Node, K.; Koretsune, Y.; Komamura, K.; Funaya, H.; Kuzuya, T.; Hori, M. Elevation of plasma adenosine levels may attenuate the severity of chronic heart failure. Cardiovasc. Drugs Ther., 1998,12(3), 307-9.

[28] Sollevi, A. Cardiovascular Effects of adenosine in man; possible clinical implications. Prog. Neurobiol., 1986, 27, 319-349.

[29] DeJong, J.W. Diagnosis of Ischemic Heart Disease with AMP Catabolites. Boston, U.S.A.: Martinus Nijhoff Publisher, 1988.

[30] Round, S.; Hsieh, L.; Agarwal, K. Effects of endotoxin injury on endothelial cell adenosine metabolism. J. Lab. Clin. Med., 1994, 123, 309-317.

[31] Yeung, P.K.; Dauphinee, J.; Gouzoules, T.; Simonsonm K.; Schindler, C. Exercise improves hemodynamic profiles and increases red blood cell concentrations of purine nucleotides in a rodent model. Ther. Adv. Cardiovasc. Dis., 2010, 4(6), 341-7.

[32] Yeung, P.K.F.; Mosher, S.J.; MacRae, D.A.; Klassen, G.A. Effect of diltiazem and its metabolites on the uptake of adenosine in blood: An in-vitro investigation. J. Pharm. Pharmacol., 1991, 43, 685-689.

[33] Yeung, P.; Mosher, S.; Li, R.; Farmer, P.S.; Klassen, G.A.; Pollak, P.T.; McMullen, M.; Ferrier, G. Erythrocyte adenosine transport: A rapid screening test for cardiovascular drugs. J. Pharmacol. Meth., 1993, 30, 163-167.

[34] Yeung, P.; Feng, J. Potential surrogate markers for pharmacodynamics of diltiazem: RBC concentrations of adenosine and adenine nucleotides. Pharm. Sci. Suppl., 1998, 1, S-329

[35] Yeung, P.; Dauphinee, J.; Simonson, K.; Gouzoules, T. RBC concentrations of ATP as potential in vivo biomarkers for cardiovascular safety of anti-hypertensive agents in rats. Clin. Pharmacol. Ther., 2009, 85(Suppl 1) PIII-8, S70.

[36] Yeung, P.; Ding, L.; Casley, W. HPLC assay with UV detection for determination of RBC purine nucleotides concentrations and application for biomarker study in vivo. J. Pharm. Biomed. Anal., 2008, 47(2), 377-382.

[37] Klein, L.C.; Yeung, P.; Berman, J.N. Cladribine inhibits a diltiazem-induced increase in red blood cell purine nucleotide concentrations in a zebrafish model. Biomarkers, 2009, 14, 554-559.

[38] Yeung, P.; Alcos, A.; Tang, J.; Casley, W. Hemodynamic effects of diltiazem in spontaneously hypertensive rats vs normotensive rats following multiple doses in vivo. Curr. Top. Pharmacol., 2008, 12(I), 39-44.

[39] Tsui, B.C.H.; Mosher, S.J.; Yeung, P.K.F. A reliable technique for chronic carotid arterial catherization in the rat. J. Pharmacol. Meth., 1991, 25, 343-352.

[40] Yeung, P.; Tang, J.; Alcos, A. Hemodynamic effects of diltiazem, losartan, amlodipine and dipyridamole in a rat model following multiple doses in vivo. Curr. Top. Pharmacol., 2009, 13(1), 41-46.

[41] Tsui, B.C.; Feng, J.D.; Yeung, P.K. Pharmacokinetics and haemodynamic effect of diltiazem in rats: effect of route of administration. J. Pharm. Pharmacol., 1998, 50(2), 183-8.

[42] Chiao, J.J.; Roberts, J.P.; Illner, H.P.; Shires, G.T. Permeability of red-cell membrane to adenosine triphosphate (ATP) molecules during hemorrhagic shock. Surgery, 1987, 102(3), 528-33.

[43] Copsel, S.; Garcia, C.; Diez, F.; Vermeulem, M.; Baldi, A.; Bianciotti, L.G.; Russel, F.G.; Shayo, C.; Davio, C. Multidrug resistance protein 4 (MRP4/ABCC4) regulates cAMP cellular levels and controls human leukemia cell proliferation and differentiation. J. Biol. Chem., 2011, 286(9), 6979-88. 
[44] Endres, C.J.; Moss, A.M.; Ke, B.; Govindarajan, R.; Choi, D.S.; Messing, R.O.; Unadkat, J.D. The role of the equilibrative nucleoside transporter 1 (ENT1) in transport and metabolism of ribavirin by human and wild-type or Ent1-/- mouse erythrocytes. J. Pharmacol. Exp. Ther., 2009, 329(1), 387-98.

[45] Kock, K.; Grube, M.; Jedlitschky, G.; Oevermann, L.; Siegmund, W.; Ritter, C.A.; Kroemer, H.K. Expression of Adenosine Triphosphate-Binding Cassette (ABC) Drug Transporters in Peripheral Blood Cells: Relevance for Physiology and Pharmacotherapy. Clin. Pharmacokinet., 2007, 46(6), 449-70.

[46] Sassi, Y.; Lipskaia, L.; Vandecasteele, G.; Nikolaev, V.O.; Hatem, S.N.; Cohen, A.F.; Russel, F.G; Mougenot, N.; Vrignaud, C.; Lechat, P.; Lompré, A.M.; Hulot, J.S. Multidrug resistanceassociated protein 4 regulates cAMP-dependent signaling pathways and controls human and rat SMC proliferation. J. Clin. Invest., 2008, 118(8), 2747-57.
[47] Bergfeld, G.R.; Forrester, T. Release of ATP from human erythrocytes in response to a brief period of hypoxia and hypercapnia. Cardiovasc. Res., 1992, 26, 40-47.

[48] Watts, J.A. Protection of ischemic hearts by $\mathrm{Ca} 2+$ antagonists. $J$. Mol. Cell Cardiol., 1986,18(1), 71-75.

[49] Rygier, L.; Yeung, P.; Berman, J. Cladribine inhibits a diltiazeminduced increase in RBC purine nucleotide concentrations in a zebrafish model. Biomarkers, 2009, (Accepted).

[50] Isaacks, R.E.; Kim, H.D. Erythrocyte phosphate composition and osmotic fragility in the Australian lungfish, Neoceratodus fosteri, and osteoglossid, Scleropages schneichardti. Comp. Biochem. Physiol. A Comp. Physiol., 1984, 79(4), 667-71.

[51] Ellsworth, M.L. The red blood cell as an oxygen sensor: what is the evidence? Acta Physiol. Scand., 2000, 168(4), 551-9.

[52] Jensen, F.B. The dual roles of red blood cells in tissue oxygen delivery: oxygen carriers and regulators of local blood flow. J. Exp. Biol., 2009, 212(Pt 21), 3387-93.

(C) Yeung et al.; Licensee Bentham Open.

This is an open access article licensed under the terms of the Creative Commons Attribution Non-Commercial License (http://creativecommons.org/licenses/by-nc/3.0/) which permits unrestricted, non-commercial use, distribution and reproduction in any medium, provided the work is properly cited. 